\title{
Water Dynamics on the Surface of the Protein Barstar
}

\author{
María Carmen Morón
}

Instituto de Ciencia de Materiales de Aragón (ICMA), Consejo Superior de Investigaciones Cientificas-Universidad de Zaragoza, Departamento de Física de la Materia Condensada, Facultad de Ciencias, Pedro Cerbuna 12, E-50009 Zaragoza, Spain.E-mail:nina@unizar.es

\section{Corresponding author:}

María-Carmen Morón

Instituto de Ciencia de Materiales de Aragón

Universidad de Zaragoza

Pedro Cerbuna 12

E-50009 Zaragoza

Spain

E-mail: nina@unizar.es

E-mail: oenola@gmail.com 


\begin{abstract}
Water plays a fundamental function in life and technology. To gain a deeper knowledge to the problem of the hydration of biomolecules, the dynamics of water around a 89residue protein of interest in molecular recognition, cancer cell research and amyloid fribrils investigations is analyzed. The biomolecule is the ribonuclease inhibitor barstar wild-type. The dynamics of the protein and the 7430 water molecules of the bath in which is immersed, is monitored during a period of $7 \mathrm{~ns}$ by using all-atom molecular dynamics simulations. The results confirm the existence of multiple time scales in the dynamics of water $\left(10^{-1}\right.$ to $\left.10^{3} \mathrm{ps}\right)$ at atomic level. That heterogeneity of residence times is not lost if the system of reference is just one atom of the inhibitor. The dehydration process of barstar is considered through the analysis of time correlation functions obtaining an averaged decay time of $\tau=84.0 \pm 0.3$ ps. A power law distribution, with scaling exponent $\alpha=0.57 \pm 0.04$, suggests that this hydration water exhibits a scale free dynamics (with respect to the residence time of solvent molecules). Most of the water molecules located on the surface of the protein spend times smaller than the picosecond while only about $1 \%$ of them stay for periods of time on the nanosecond scale.
\end{abstract}




\section{Introduction}

Water plays a fundamental function in life. Exhibiting a ubiquitous character, biomolecules like proteins, nucleic acids, membranes etc, are immersed in it. Nevertheless it should not be regarded as just a solvent. Investigating the biochemical and physicochemical properties of water is essential for understanding biological and medical phenomena. ${ }^{1}$ Most of the biological molecules are physiologically inactive without the presence of water. It plays an active role mediating molecular reactions: electron and proton transfer, enzymatic catalysis, protein folding, DNA repair and replication, molecular recognition between proteins etc. ${ }^{2}$ Detailed information of the dynamics of water localized to a biomolecule surface is necessary to understand many aspects of biological activity.,

Different experimental techniques have been used to study the dynamics of biomolecules in water solution: neutron scattering, dielectric relaxation, nuclear magnetic resonance spectroscopy, time-resolved fluorescence ... . ${ }^{5,6}$ All these different techniques detect a range of time scales which are difficult to assign to particular decay channels. $^{3,7}$ The reason is the intricate nature of the dynamics of the different components of the system (water, biomolecule, counterions...) which interrelate in a complex manner. It is in that sense that molecular dynamics simulations can help making possible the identification and assignment of those various relaxation processes. Solving the equations of motion for each atom in particular, they can provide a microscopic and at the same time quantitative view of the problem. ${ }^{8}$ Untangling those different contributions, simulations can be regarded as essential to improve the knowledge of those experimental time scales.

There are numerous studies, experimental as well as theoretical, showing that water dynamics near a protein surface is only affected to a small extent, typically a 2-4-fold slowdown. ${ }^{6,9-11}$ Simultaneously there are some other works, using experimental techniques and computer simulations, which report a wide range of time scales for the dynamics of water around biomolecules from femto- to nanoseconds $\left(10^{-15}\right.$ to $\left.10^{-9} \mathrm{~s}\right)$. In particular, they describe a slow component of the solvation dynamics on the 1001000 picoseconds time scale., $2-14$ These values are 2-3 orders of magnitude larger when compared with bulk water. ${ }^{4,14}$ Those apparent contradictions between different reports have been the object of a continuous debate. ${ }^{4,15}$ Slow hydration water is interesting for several reasons. Firstly, slow solvation retards charge transfer processes. $^{2,3}$ This discovery is essential in polar reactions occurring in supramolecular assemblies. In fact, the rate of a chemical reaction is inversely proportional to the solvent time decay. ${ }^{16,17}$ Secondly, a slower hydration water aids to maintain the structure of a protein under unfavourable circumstances, preventing dehydration etc. ${ }^{2}$ Thirdly, water is the lubricant of life. Slow solvation increases friction on the surface involved, even at the spatial scale this work concerns: the nano-scale. ${ }^{18}$

Clearly a better understanding of solvation dynamics of complex systems is desirable. ${ }^{4,19}$ In this paper we present a study of the dynamics of the first hydration shell of the biomolecule barstar through the analysis of the residence times of the water molecules on it. Barstar, which can be found in Bacillus Amyloliquefaciens as a ribonuclease inhibitor, is a 89-residue protein of interest in molecular recognition (drug discovery $)^{20}$, cancer cell research ${ }^{21}$ and amyloid fibrils investigations 
(neurodegenerative diseases) $^{22}$. To gain an atomic level knowledge we use all-atom molecular dynamics simulations to follow the dynamics of the system (details are presented in section II of the paper). Thus in section IIIa (macroscopic view), an averaged relaxation time is calculated through a correlation function. In section IIIb a power low distribution suggests the scale free nature of the hydration water considered, and in section IIIc a microscopic analysis (a unique atom of the protein is considered) shows the existence of various time scales in the water surrounding barstar. Spatial and temporal scales involved in this work: nano and femto-nano scales, respectively.

\section{Computational Details}

We have performed classical all-atom molecular dynamics simulations of aqueous solutions of the protein barstar (wild type). The initial structure of this inhibitor was obtained from the Protein Data Bank (PDB) code 1brs. We did not keep the water molecules included in the crystallographic PDB data. The simulations were conducted using a double precision version of the GROMACS software package. ${ }^{23,24}$ After adding the hydrogens, the whole protein was immersed in a large cubic box of well equilibrated water by carefully avoiding unfavorable contacts. The box dimensions were chosen to ensure a $1 \mathrm{~nm}$ thick solvation shell around the protein structure. This procedure resulted in a solvated starting structure containing 23728 atoms which include 1435 protein atoms and 7430 water molecules. This system was subjected to a series of steepest descent and conjugate gradient energy minimizations to remove possible unfavorable interactions between solute and solvent.

The molecular dynamics simulations were performed by integrating the equations of motion employing stochastic dynamics (Langevin) and a time step (resolution) of $2 \mathrm{fs}$ $(0.002 \mathrm{ps})$. A multi-component all-atom force field (OPLS-AA) described the interactions between the constituents of the system. ${ }^{25,26}$ Consistent with this force field, the three site simple point charge model (SPC) ${ }^{27}$ was used for modeling water. After an equilibration period, the trajectory for data production was performed in the canonical ensemble, i. e. at constant $\mathrm{N}, \mathrm{V}$ and $\mathrm{T}$, amounting a total time of $7 \mathrm{~ns}$ in order to capture the slow dynamics of the solvent. The simulations were carried out at $300 \mathrm{~K}$ in a simulation box of $6.2 \mathrm{~nm}$ along the three axes. We adopt the periodic boundary condition to prevent boundary effects. During the production run the root mean square deviation of the barstar molecule was found to maintain a steady value (around 0.17 $\mathrm{nm})$ with respect to its initial configuration indicating an equilibrated state. The cutoff length for the Lenard-Jones and Coulomb potentials was set at $1.4 \mathrm{~nm}$. All bond lengths were constrained using the LINCS algorithm. ${ }^{28}$ The NVT production run was saved every $0.1 \mathrm{ps}$ for later analysis.

An equivalent simulation was performed (same conditions), but this time only water molecules were considered (bulk water). As a result, the trajectory of a total of 7918 solvent molecules was monitored during a production run of 7 nanoseconds which was saved every 0.2 ps for later analysis. 


\section{Results and Discussion}

\section{IIIa. Multiple Time Scales: Macroscopic view}

The dynamics of the hydration water can be described by evaluating, as a function of time, the occupation of the water molecules on different shells around the protein. That occupancy is related to the mobility (diffusion coefficient) of the water molecules through the shell considered. Indeed, the dynamical properties of a fluid in the solvation shells of a solute can be obtained from time correlation functions. ${ }^{29}$ Thus, we have considered a normalized occupation function defined as

$$
\mathrm{C}(\mathrm{t})=\sum_{i=1}^{N}[<\mathrm{Oi}(\text { to }) \mathrm{Oi}(\text { to }+t)>/<\mathrm{Oi}(\text { to }) \mathrm{Oi}(\text { to })>]
$$

where $\operatorname{Oi}\left(\mathrm{t}_{\mathrm{o}}+\mathrm{t}\right)$ takes the value of 1 (unity) if the water molecule $\mathrm{i}$, that is within the region of interest at the time origin $t_{0}$, still exists in that region at time $t_{0}+t$ (the solvent molecule is not required to remain in that region at all times between $t_{0}$ and $t$ ). Otherwise $\mathrm{Oi}\left(\mathrm{t}_{\mathrm{o}}+\mathrm{t}\right)$ equals $0 . \mathrm{N}$ is the total number of water molecules within the shell considered at $t_{0}$. The angular brackets denote averaging over various time origins in order to take into account different areas of the trajectory as well as improve statistics. Therefore $\mathrm{C}(\mathrm{t})$ gives the average number of water molecules that still remain, in the region of interest, after a time $t$. In order to analyse the first hydration layer around the protein, we have calculated that function for water molecules that are present within a shell of thickness $0.4 \mathrm{~nm}$ from all atoms of the biomolecule, hydrogen excluded. We chose $0.4 \mathrm{~nm}$ because although the number of water molecules will be comparatively small, larger radius will result in taking into account more of the bulk dynamics.

The function $\mathrm{C}(\mathrm{t})$ was calculated, from the molecular dynamics simulations, following Equation 1 with a resolution of 0.1 ps by averaging over 14 initial times which were homogeneously distributed along the whole simulation (Fig. 1 upper curve). Increasing the number of time-origins would improve precision (statistics) which is already good enough. The tendency of the curve consists of a fast initial decay followed by a slower one. This trend can not be described by a single exponential law. A fit of these relaxation data allow to successfully depict the $\mathrm{C}(\mathrm{t})$ time evolution using a stretched exponential function $\exp \left[-(\mathrm{t} / \tau)^{\mathrm{b}}\right] .^{30}$ The stretched exponential decay, also known as the KWW (Kohlraush-Williams-Watts) function, is often observed in several phenomena in complex condensed matter systems and supercooled liquids. ${ }^{31}$ The fit of our data to that function supplies parameter values $\tau=84.0 \pm 0.3$ ps and $\beta=0.48^{ \pm} 0.01$.

The time constant $\tau=84.0 \pm 0.3$ ps gives an estimation of the permanence time of waters in the solvent shell under study, providing the overall time scale over which the process develops. A relationship between the occupation time and solvation dynamics can be intuitively contemplated since that occupation time is a measurement of the mobility of the water molecules. Solvation on a similar time scale has been reported for water molecules confined at the surface of different proteins ${ }^{32}$, micelles ${ }^{33}$, nanoscopic non polar solutes ${ }^{29}$ and other biomolecules and hydrophobic materials ${ }^{12,34}$, both from experiments and simulations. The normalized occupation function $\mathrm{C}(\mathrm{t})$, Equation 1, was also calculated for a $0.4 \mathrm{~nm}$ thick shell of bulk water with inner radius corresponding to the radius of barstar (Fig. 1 lower curve). The diameter of the protein 
can be estimated as $3.1 \pm 0.5 \mathrm{~nm}$ (Protein Data Bank code 1brs). The function $\mathrm{C}(\mathrm{t})$ was calculated by averaging over 14 initial times homogeneously distributed along the whole simulation of neat solvent. The fit to a stretched exponential function provides a decay time of $36^{ \pm} 1$ ps. However this result for bulk water should be considered with precaution due to the simplifications of the model and the quality of the fit. Thus, there are solvent molecules on both walls of the shell (inner and outer). In a first approximation it might be guessed that this fact would reduce the relaxation time about twofold. In addition the time scales of rotational and translational motions for bulk water have been reported to range from a few tens of femtoseconds to a few tens of picoseconds. ${ }^{14,35}$ Therefore the decay time obtained for the inhibitor barstar would be in agreement with the studies reporting that water dynamics near a protein surface is affected to a small extend (2-4-fold slowdown). ${ }^{6,9-11}$ On the other hand, recent experiments on solvation dynamics, performed on native barstar and monitored by the time-dependent dynamics Stokes shift measurements, detected mean solvent relaxation times within the range $10-100 \mathrm{ps}^{36}$

The exponent of the KWW exponential supplies information about how large is the deviation of the relaxation curve from a classical exponential since for $\beta=1$ the mathematical expression of a simple exponential is restored. In our case, a beta value of $0.48 \pm 0.01$ shows a large deviation from a classical exponential decay indicating the presence of significant temporal disorder in the system. ${ }^{29}$ Stretched exponentials are frequently employed for modelling phenomena characterized by multiple relaxation rates. ${ }^{37}$ Stretched exponents $\beta \sim 0.5$ were described for other proteins as lysozyme ${ }^{38}$ and for diffusion-controlled processes in molecular luminiscence ${ }^{39}$.

\section{IIIb. Multiple Time Scales: Mesoscopic view}

Since the appearance of stretched exponentials can be related to the spread in the permanence time values indicating intricate time scales ${ }^{40}$, the number of water molecules that spend a given time $t$ within the already considered cutoff of $0.4 \mathrm{~nm}$ from the protein has been calculated as $\mathrm{N}_{\mathrm{w}}(\mathrm{t})$. That number was obtained by averaging over 25 origins of time distributed along the whole trajectory of the molecular simulation. Most of the water molecules stay during short periods of time near the protein, while few of them spend much longer times, reflecting heterogeneity in the temporal behaviour of hydration water.

If the evolution of $\mathrm{N}_{\mathrm{w}}(\mathrm{t})$ with time is represented in a double-logarithm plot, a straight line is obtained (see Fig. 2). This result shows that the distribution of the water molecules within the first hydration layer (cutoff $0.4 \mathrm{~nm}$ ) of the protein barstar follows a power law, $\mathrm{N}_{\mathrm{w}} \sim \mathrm{t}^{-}{ }^{\circ}$, as a function of the residence times in that shell. Therefore that biological water displays the same type of distribution at any scale, i.e., it is scale free ${ }^{41}$. Some empirically observed networks have been reported as scale free: social, computer or semantic networks. ${ }^{41}$ In our case, a scale free distribution in time with the agents at atomic level (water molecules) is presented.

In the case of the hydration water of barstar the scaling exponent was calculated as $\alpha=$ $0.57 \pm 0.04$. Power law distributions with $1 / 2<a<3 / 2$ were found for diffusion models of ion-channel gating in proteins. ${ }^{42}$ In our case we could define a binary signal 
in time of either inside or outside the cutoff of $0.4 \mathrm{~nm}$. The position $\mathrm{x}=0.4 \mathrm{~nm}$, where $\mathrm{x}$ is the distance of the water molecule under consideration to the protein, would correspond to the open-channel state, and the remaining positions $\mathrm{x}<0.4 \mathrm{~nm}$ would correspond to the closed-channel states.

\section{IIIc. Multiple Time Scales: Microscopic view}

To gain a microscopic understanding of the observed scale free dynamics, we calculated the distribution of times that the water molecules exhibit around a given environment (atom) of barstar. Thus, the time that the solvent molecules spend at a distance smaller than $0.4 \mathrm{~nm}$ from the nitrogen atom of the amine group of the non polar residue Tryptophan 44 is shown in Fig. 3. More than a biphasic behaviour, as reported from macroscopic studies for various biomolecules ${ }^{3}$, a wide distribution of times is depicted. This figure provides an atomistic view for the heterogeneity of times detected by the residence time power law distribution (Fig. 2) and the stretched exponential ( $\beta$ departure from 1, Fig. 1). Similar calculations to those showed in Fig. 3 performed for more than 50 different sites distributed along the whole protein, confirm the multi-time dynamics at atomistic level of the first hydration shell around barstar. Since the lack of homogeneity associated to the non-exponential relaxation might be correlated with a spread in the barriers of the energy landscape, ${ }^{43,44}$ and the singular properties of water are widely due to the peculiar character of its hydrogen-bonded network, we have carried out an study of the hydrogen bonds that different water molecules exhibit with barstar and, on the other hand, with the rest of the solvent. Thus, we have selected three types of water molecules spending around $1 \mathrm{~ns}, 100 \mathrm{ps}$ and a few ps, respectively, in the first hydration layer of the protein. We used standard geometrical criteria for the determination of the existence of an hydrogen bond between two atoms: the distance between donor and acceptor atoms within $0.35 \mathrm{~nm}$ and the hydrogen-donor-acceptor angle smaller than $30^{\circ} .^{45}$

We have found that the water molecules that stay longer periods of time near the protein barstar, present more hydrogen bonds with atoms of the biomolecule than with the rest of the solvent, and vice versa. Let us consider a water molecule (label 835 in Fig. 3) which spends more than $1 \mathrm{~ns}$ in the first hydration shell of 7 residues of the protein barstar. Four of them are non polar (Alanine 40, Leucine 41, Valine 45 and Tryptophan 44) while the rest are polar residues with no electric charge (Threonine 42, Glycine 43 and Tyrosine 47). This water molecule exhibits, during this period of time, an average of 2.5 hydrogen bonds with the inhibitor but just 1 with the solvent. Each bulk water molecule in solution is able to form 4 hydrogen bonds at room temperature with an average of $\sim 3.5 .^{5,46}$ If that calculation is performed for a water molecule which spend just some few picoseconds within the first solvation layer of those residues, the average decreases to 0 hydrogen bonds with barstar and increases to 3.5 ones with the solvent. It is important to remember that a water-protein hydrogen bond is stronger than a waterwater one. $^{47,48}$ This fact contributes to the preference of the water molecules for, if possible, being hydrogen bonded to the protein. Roy and Bagchi have recently studied the free energy barriers for escape of water molecules from protein hydration layer. ${ }^{35}$ They broadly classify water molecules at the protein surface as free and quasi-bound species. Interfacial free water molecules do not form any hydrogen bonds with the protein while interfacial quasi-bound water molecules can be either singly or double hydrogen bonded. They have found that water molecules double hydrogen bonded to 
the protein HP-36 exhibit longer residence times than those singly hydrogen bonded and much longer times than interfacial free water molecules. Construction of the waterprotein hydrogen bonds by suppression of the water-water ones prevents freezing of water around the biomolecule. This quality of the hydration water preserves life at low temperatures. ${ }^{49-51}$

Up to now this work has been devoted to time resolved dynamics. In this paragraph we are going to address some consideration to spatially resolved dynamics. Thus, fluorescence experiments have shown that the dynamics of hydration water changes from one to another region of biomolecules and other organized assemblies. ${ }^{2}$ However, it is not always easy to disentangling contributions of different processes. For example, the fluorescence relaxations observed does not necessarily reflect solvation dynamics only. ${ }^{2}$ A comparison between the landscapes depicted in Fig. 3 and 4, illustrates the dependence of the residence time distribution with the particular environment of barstar. The sites of reference considered in these figures are different: the oxygen of the carboxyl group on the polar positively charged residue Lysine 22 and the nitrogen atom of the amine group of the non polar Tryptophan 44 residue of the biomolecule, respectively. Similar calculations to those showed in these figures performed for more than 50 different sites of the protein confirms, at atomic level, that the landscape of the time distribution is highly dependent on the nature of the environment considered, i.e., site specific. The solvation dynamics, even from an atomistic point of view, vary notably from one site to another on the surface of the protein barstar. The topological disorder of the biomolecule, mainly due to its intrinsic chemical nature, clearly influences this spatial heterogeneity. This result is in good agreement with fluorescence experiments and simulation data ${ }^{2}$ but also with the existence of different relaxation times for different regions of proteins ${ }^{52}$.

Some water molecules in the first hydration layer of barstar display survival times around or even larger than 1 ns (Fig. 3). Due to the significance of slow dynamics in hydration water, the average number of these slower water molecules around the protein was calculated giving a value of $5 \pm 1$ molecules. This is a relatively small value $(\sim 1 \%)$ when compared with $370 \pm 5$, the total number of water molecules within the referred hydration layer (cutoff $0.4 \mathrm{~nm}$ ). These water molecules that are restricted in motion constitute a bridged structure by simultaneously forming hydrogen bonds with various residues of barstar (see above). The rarity of water molecules doubly hydrogen bonded to the protein HP-36 has been reported to be due to the fact that their formation requires constrained arrangement which is entropically demanding. ${ }^{35}$ Relaxation times in the nanosecond scale have been described in various works performed on biomolecules. ${ }^{2}$ These water molecules with long residence times, contribute to increase the mean value of the residence time of the solvent molecules in the first hydration shell. Moreover, the friction on the protein surface due to solvation should be intuitively influenced by the mean time that the water molecules spend in the hydration shell of the biomolecule without being exchanged with solvent. Consequently due to these slower molecules the friction on the protein due to the hydration layer, a nanoscale friction, increases.

However, most of the water molecules of the first hydration shell of barstar exhibit residence times which are orders of magnitude smaller than the nanosecond (see Figs. 2, 3 and 4). We are talking about a scale of time smaller than the picosecond. Timedependent dynamics Stokes shift (TDSS) measurements, performed on proteins in 
solution, detect a fast component ( $<1$ ps $)$, which is popularly assigned to the relaxation of the bulk solvent, and slower components ( $>10 \mathrm{ps}$ ) which are assigned to the hydration water. ${ }^{18,47,53}$ The small residence times detected for the hydration water of barstar from atomic level analysis (see above) show that this kind of water can also contribute to the fast solvent dynamics detected for that protein from recent TDSS experiments $^{36}$. That is, not only the bulk water but also the hydration one can be responsible for the fast dynamics exhibited by TDSS measurements performed on this system. In addition it is possible to say that most of the water molecules in the first hydration shell around barstar display weak links with the protein (they do not spend much time near it). In this sense, there are far more weak links than strong ones around barstar. The reason is the scale free nature of that hydration water. Weak links are responsible for the fast dynamics exhibited by the referred hydration water. Strong links are important for complex systems but weak links are essential to stabilize them. ${ }^{54}$

The atomic level analysis performed in this section IIIc shows that the dynamics of the hydration water around that protein exhibits residence times which are 2-3 orders of magnitude larger than that of bulk water. However, the results obtained in section IIIa (decay time) suggest than that hydration water would be affected to a smaller extent. Both apparent contradictory results could be conciliated considering: i) that the calculated relaxation time represent an average over a wide range of time scales, and ii) that only around $1 \%$ of the hydration water surrounding barstar exhibits residence times on the nanosecond scale. The absence of any noticeable slow component in NMR relaxation experiments have been recently explained as due to the average time constant this technique provides. ${ }^{4,55}$ Then, if macroscopic data are considered (global/averaged view), the dynamics of the hydration water would be affected only to a small extent (24-fold slowdown) due to the reduced number of very slow solvent molecules in such a layer. However if microscopic data are taken into account, the hydration water dynamics would exhibit a slow component neatly smaller than that of the bulk water (23 orders of magnitude). 


\section{Conclusion}

The study of the hydration water of barstar exhibits a broad distribution of relaxation times suggesting a complex landscape for the flow out of the dynamics of this special water. Residence time distribution functions, calculated for the entire solvent molecules of the first hydration shell, reveal that such temporal distribution follows a power law. Therefore that hydration water around the protein can be regarded as a scale free system with respect to the distribution of residence times of water molecules. In this sense, most of the solvent molecules located on the surface of the protein (cutoff $0.4 \mathrm{~nm}$ ) spend times smaller than the picosecond on it while only about $1 \%$ of them stay for periods of time on the nano second scale. There are far more weak links than strong ones around the biomolecule. The microscopic analysis of the residence times shows that the hydration water around barstar can also contribute to the fast solvent dynamics detected for that protein from recent time-dependent dynamics Stokes shift experiments. That is, not only the bulk water but also the solvent molecules located on the surface of barstar can be responsible for the fast dynamics exhibited by those experimental measurements.

In this article it is show how a better understanding of the dynamics of water surrounding proteins may benefits from atomistic deeper approaches. It would be of interest to analyse if the hydration water of other biomolecules or materials like polymers, nanotubes, nanoassemblers etc. can be described as a scale free system. We look forward to having contributed in some way to a better understanding of the dynamics of this type of always fascinating water.

Acknowledgements. Dr. Diego Prada-Gracia and Dr. Fernando Falo are fully acknowledged for inestimable discussions, invaluable introduction to the GROMACS software package and an always excellent disposition. Dr. Pedro J. Martínez and Dr. Diego Prada-Gracia are also acknowledged for the management and maintenance of the computer cluster where the simulations were performed. This work was funded by the Spanish MICINN (Ministerio de Ciencia e Innovación) and the FEDER (ERDFEuropean Regional Development Fund) through Projects No. MAT2007-61621 and FIS2011-25167. 


\section{References}

1. S. K. Kim, T. Ha and J. P. Schermann, Phys. Chem. Chem. Phys., 2010, 12, 10145-10146.

2. K. Bhattacharyya, Chem. Commun. 2008, 25, 2848-2857.

3. B. Bagchi B and B. Jana, Chem. Soc. Rev., 2010, 39,1936-1954.

4. B. Bagchi, Chem. Phys. Lett., 2012, 529, 1-9.

5. T. M. Raschke, Curr. Opin. Struc. Biol., 2006, 16, 152-159.

6. K. E. Furse and S.A. Corcelli, J. Phys. Chem. Lett., 2010, 1, 1813-1820.

7. D. Zhong, S. K. Pal and A.H. Zewail, Chem. Phys. Lett., 2011, 503, 1-11.

8. M. Vogel, J. Phys. Chem. 2009, 113, 9386-9392.

9. B. Halle and L. Nilsson, J. Phys. Chem. B, 2009, 113, 8210-8213.

10. A. A. Golosov and M. Karplus, J. Phys. Chem. B, 2007, 111, 1482-1490.

11. L. Nilsson and B. Halle, Proc. Natl. Acad. Sci. USA, 2005, 102, 13867-13872.

12. K. Sahu, S. K. Mondal, S. Ghosh and K. Bhattacharyya, Bull. Chem. Soc. Jpn., 2007, 80, 1033-1043.

13. K. Bhattacharyya, Acc. Chem. Res., 2003, 36, 95-101.

14. K. Sahu, S.K. Mondal, S. Ghosh, D. Roy and K. Bhattacharyya, J. Chem. Phys. 2006, 124, 124909-7.

15. T. Li, A. A. Hassanali, Y. T. Kao, D. Zhong and S. J. Singer, J. Am. Chem. Soc., 2007, 129, 3376-3382.

16. G. van der Zwan and J. T. Hynes, J .Phys. Chem., 1985, 89, 4181-4188.

17. G. van der Zwan, J. T. Hynes, Chem. Phys., 1984, 90, 21-35.

18. S. K. Pal, J. Peon, B. Bagchi and A. H. Zewail, J. Phys. Chem. B, 2002, 106, 12376-12395.

19. N. Giovambattista, P. J. Rossky and P. G. Debenedetti, Annu. Rev. Phys. Chem., 2012, 63, 179-200.

20. M. Hoefling and K. E. Gottschalk, J. Struct. Biol., 2010, 171, 52-63.

21. M. P. Nikitin, T. A. Zdobnova, S.V. Lukash, O. A. Stremovskiy and S. M. Deyev, Proc. Natl. Acad. Sci. USA, 2010, 107, 5827-5832.

22. A. Sekhar and J. B. Udgaonkar, Biochemistry 2011, 50, 805-819.

23. D. Van der Spoel, E. Lindahl, B. Hess, G. Groenhof, A. E. Mark and H. J. C. Berendsen, J. Comput. Chem., 2005, 26, 1701-1718.

24. H. J. C. Berendsen, D. van der Spoel and R. van Drunen, Comput. Phys. Commun., 1995, 91, 43-56.

25. W. L. Jorgensen, D. S. Maxwell and J. Tirado-Rives, J. Am. Chem. Soc., 1996, 118, 11225-36.

26. E. K. Watkins, W. L. Jorgensen, J. Phys. Chem. A, 2001, 105, 4118-4125.

27. H. J. C. Berendsen, J. P. M. Postma, W. F. vanGunsteren and J. Hermans, in Intermolecular Forces, ed B. Pullman, Reidel Publishing Company, Dordrecht, The Netherlands, 1991, pp 331-342.

28. B. Hess, H. Bekker, H. J. C. Berendsen and J. G. E. M.Fraaije, J. Comput. Chem., 1997, 18, 1463-1472.

29. N. Choudhury, J. Phys. Chem. C, 2007, 111, 2565-2572.

30. R. Kohlrausch, Ann. Phys. (Leipzig), 1847, 148, 353-405; G. Williams and D. C. Watts, Trans. Faraday Soc., 1970, 66, 80-85.

31. J. C. Phillips, Rep. Prog. Phys., 1996, 59, 1133-1207.

32. A. R. Bizarri and S. Cannistraro, J. Phys. Chem. B 2002, 106, 6617-6633. 
33. F. Sterpone, G. Marchetti, C. Pierleoni and M. Marchi, J. Phys. Chem. B 2006, 110, 11504-11510.

34. N. J. Choudhury, Chem. Phys., 2010, 132, 064505.

35. S. Roy and B. Bagchi, J. Phys. Chem. B, 2012, 116, 2958-2968.

36. A. Jha, K. Ishii, J. B. Udgaonkar, T. Tahara and G. Krishnamoorthy, Biochemistry, 2011, 50, 397-408.

37. V. A. Makarov, B. K. Andrews, P. E. Smith and B. M. Pettit, Biophys. J., 2000, 76, 2966-2974.

38. J. Servantie, G. Atilgan and A. R. Atilgan, J. Chem. Phys., 2010, 133, 085101.

39. S. A. Rice, in Comprehensive Chemical Kinetics, ed C. H. Bamford, C. F. H. Tipper and R. G. Compton, Elsevier, Amsterdam, 1985, vol 25.

40. R. G. Palmer, D. L. Stein, E. Abrahams and P. W. Anderson, Phys. Rev. Lett., 1984, 53, 958-961.

41. R. Albert and A. L. Barabasi, Rev. Mod. Phys., 2002, 74, 47-97.

42. G. L. Millhauser, E. E. Salpeter and R. E. Oswald, Proc. Natl. Acad. Sci. USA, 1988, 85, 1503-1507.

43. S. Sastry, P. G. Debenedetti and F.H. Stillinger, Nature 1998, 393, 554-557.

44. F. H. Stillinger, Science, 1995, 267, 1935-1939.

45. M. Jana and S. Bandyopadhyay, J. Chem. Phys., 2011, 134, 025103.

46. B. Bagchi, Chem. Rev. 2005, 105, 3197-3219.

47. N. Nandi and B. Bagchi, J. Phys. Chem. B, 1997, 101, 10954-10962.

48. N. Nandi, B. Bagchi, J. Phys. Chem. A, 1998, 102, 8217-8221.

49. Y. Uda, S. Zepeda, F. Kaneko, Y. Matsuura and Y. Furukawa, J. Phys. Chem. $B, 2007,111,14355-14361$.

50. Y. Yeh and R. E. Feeney, Chem. Rev., 1996, 96, 601-617.

51. J. J. Skalicky, K. D. Sukumaran, J. L. Mills and T. Szyperski, J. Am. Chem. Soc., 2000, 122, 3230-3231.

52. S. K. Sinha, S. Chakraborty and S. Bandyopadhyay, J. Phys. Chem. B, 2008, 112, 8203-8209.

53. N. Nandi, K. Bhattacharyya and B. Bagchi, Chem. Rev., 2000, 100, 2013-2046.

54. P. Csermely, Trends. Biochem. Sci., 2004, 29, 331-334.

55. B. Jana, S. Pal and B. Bagchi, J. Chem. Sci., 124, 317-325. 


\section{Figures}

Fig. 1. Normalized occupation time correlation function for water in the first hydration shell of the protein barstar (upper curve). $\mathrm{C}(\mathrm{t})$ represents the number of water molecules that remain in the region of interest at a given time $t$ with respect to that number at the initial time. The symbols are the data points obtained from molecular dynamic simulations at $300 \mathrm{~K}$. The function $\mathrm{C}(\mathrm{t})$ was calculated using Equation 1 with a cutoff of $0.4 \mathrm{~nm}$. That equation was also used to estimate $\mathrm{C}(\mathrm{t})$ for bulk water from a simulation of neat solvent (lower curve). In both cases the continuous line represents the fit using a stretched exponential function. See text for details.

Fig. 2. Number of water molecules (y-axis) that spend a time $t$ (x-axis) within a cutoff of $0.4 \mathrm{~nm}$ from the protein surface. Both axes are in logarithmic scale. The resolution in time is $0.1 \mathrm{ps}$. The number of water molecules has been obtained by averaging over 25 time origins distributed along the whole trajectory of the molecular dynamics simulation. The straight line represent the best fit to a power law distribution $t^{-\alpha}$ with $\alpha=0.57^{ \pm} 0.04$.

Fig. 3. Distribution of residence times for water molecules located within a cutoff of $0.4 \mathrm{~nm}$ from a specific site of the protein: the nitrogen atom of the amine group on the non polar Tryptophan 44 residue. If a given water molecule ( $y$-coordinate), at a particular time of the simulation ( $x$-coordinate), is located at a distance of that site smaller than the cutoff, then a cross appears at the corresponding $(x, y)$ location. If the distance is higher or equal than the cutoff, a blank is settled instead. The presence of multiple time scales can be clearly seen at this atomic level. Remark the slow dynamics exhibited by molecules with residence times on the nano-scale. Data calculated from molecular dynamics simulations. The resolution in time is $1 \mathrm{ps}$.

Fig. 4. Distribution of residence times for water molecules located within a cutoff of $0.4 \mathrm{~nm}$ from a specific site of the protein: the oxygen atom of the carboxyl group on the polar positively charged Lysine 22 residue. If a given water molecule ( $y$-coordinate), at a particular time of the simulation ( $x$-coordinate), is located at a distance of that site smaller than the cutoff, then a cross appears at the corresponding $(x, y)$ location. If the distance is higher or equal than the cutoff, a blank is settled instead. No residence times on the nanosecond scale can be observed. Data calculated from molecular dynamics simulations. The resolution in time is $1 \mathrm{ps}$. 


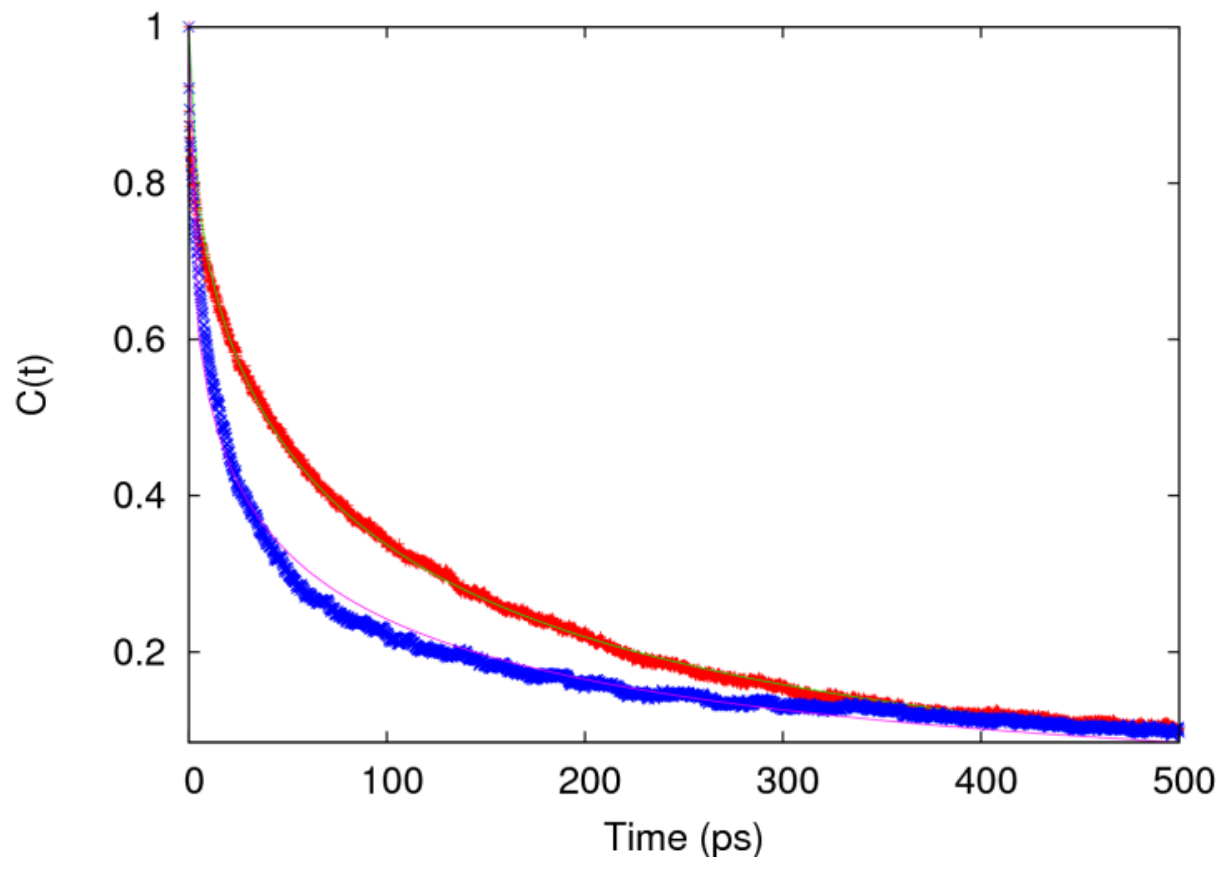

Fig. 1. Normalized occupation time correlation function for water in the first hydration shell of the protein barstar (upper curve). $\mathrm{C}(\mathrm{t})$ represents the number of water molecules that remain in the region of interest at a given time $t$ with respect to that number at the initial time. The symbols are the data points obtained from molecular dynamic simulations at $300 \mathrm{~K}$. The function $\mathrm{C}(\mathrm{t})$ was calculated using Equation 1 with a cutoff of $0.4 \mathrm{~nm}$. That equation was also used to estimate $\mathrm{C}(\mathrm{t})$ for bulk water from a simulation of neat solvent (lower curve). In both cases the continuous line represents the fit using a stretched exponential function. See text for details. 


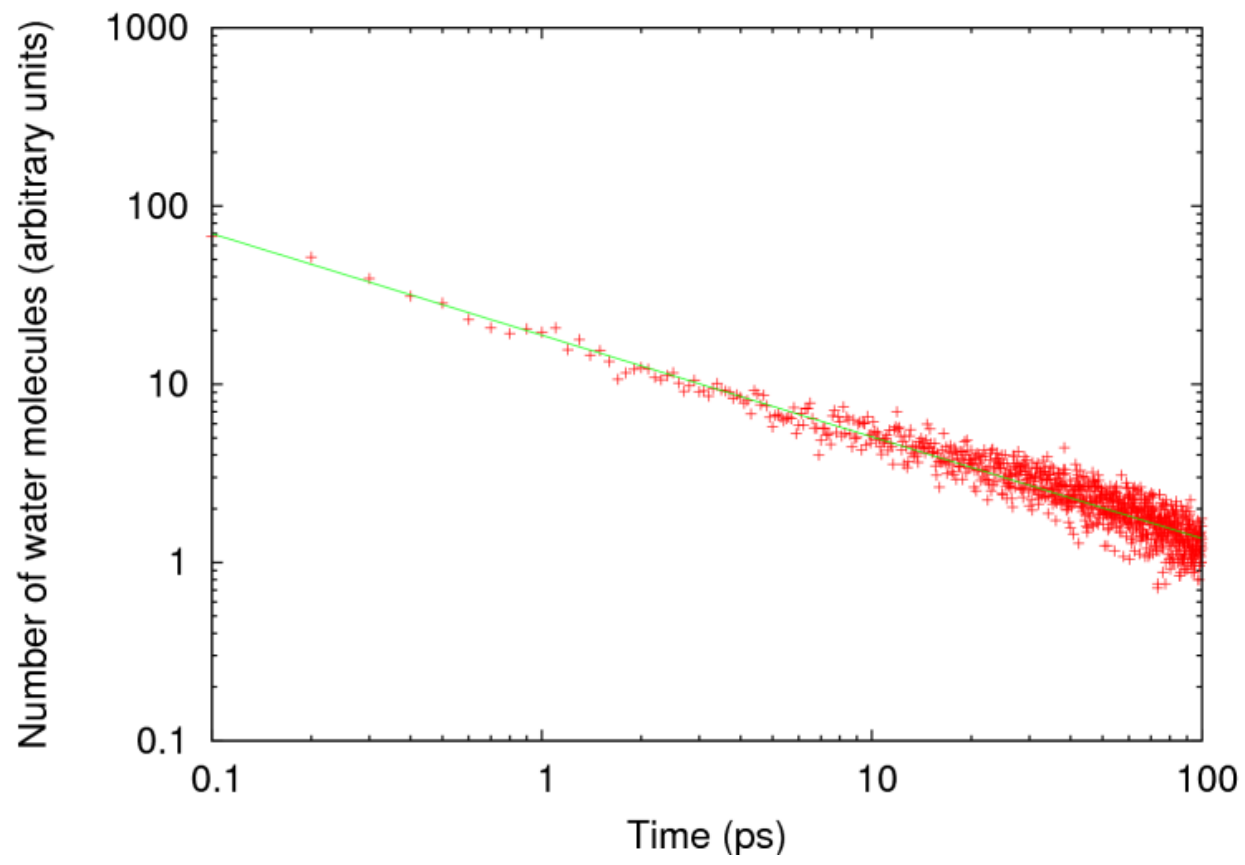

Fig. 2. Number of water molecules (y-axis) that spend a time $\mathrm{t}$ (x-axis) within a cutoff of $0.4 \mathrm{~nm}$ from the protein surface. Both axes are in logarithmic scale. The resolution in time is $0.1 \mathrm{ps}$. The number of water molecules has been obtained by averaging over 25 time origins distributed along the whole trajectory of the molecular dynamics simulation. The straight line represent the best fit to a power law distribution $\mathrm{t}^{-}{ }^{\alpha}$ with $\alpha=0.57^{ \pm} 0.04$. 


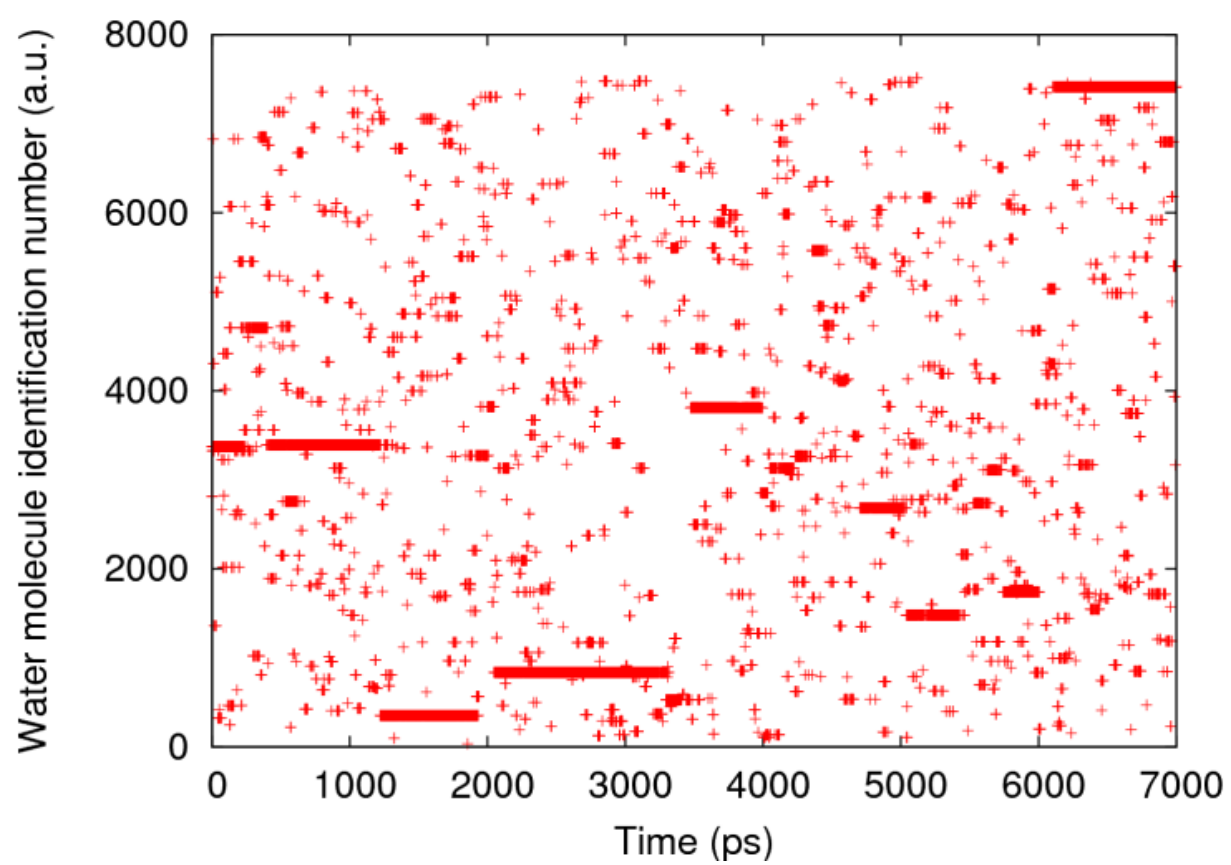

Fig. 3. Distribution of residence times for water molecules located within a cutoff of $0.4 \mathrm{~nm}$ from a specific site of the protein: the nitrogen atom of the amine group on the non polar Tryptophan 44 residue. If a given water molecule ( $y$-coordinate), at a particular time of the simulation ( $x$-coordinate), is located at a distance of that site smaller than the cutoff, then a cross appears at the corresponding $(x, y)$ location. If the distance is higher or equal than the cutoff, a blank is settled instead. The presence of multiple time scales can be clearly seen at this atomic level. Remark the slow dynamics exhibited by molecules with residence times on the nano-scale. Data calculated from molecular dynamics simulations. The resolution in time is $1 \mathrm{ps}$. 


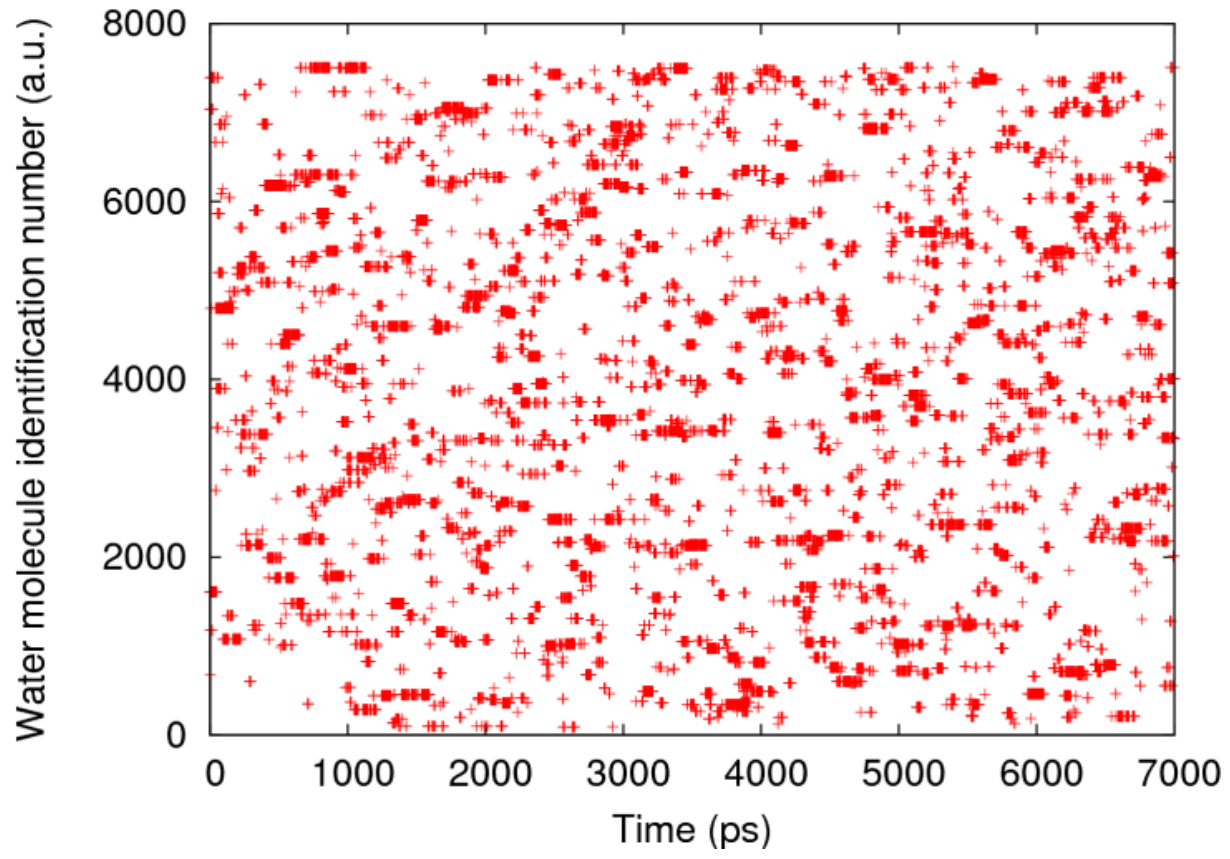

Fig. 4. Distribution of residence times for water molecules located within a cutoff of $0.4 \mathrm{~nm}$ from a specific site of the protein: the oxygen atom of the carboxyl group on the polar positively charged Lysine 22 residue. If a given water molecule ( $y$-coordinate), at a particular time of the simulation ( $x$-coordinate), is located at a distance of that site smaller than the cutoff, then a cross appears at the corresponding $(x, y)$ location. If the distance is higher or equal than the cutoff, a blank is settled instead. No residence times on the nanosecond scale can be observed. Data calculated from molecular dynamics simulations. The resolution in time is $1 \mathrm{ps}$. 